\title{
Efficient quantum computation using coherent states
}

\author{
H. Jeong and M. S. Kim \\ School of Mathematics and Physics, Queen's University, Belfast BT7 1NN, United Kingdom
}

(November 2, 2018)

\begin{abstract}
Universal quantum computation using optical coherent states is studied. A teleportation scheme for a coherent-state qubit is developed and applied to gate operations. This scheme is shown to be robust to detection inefficiency.
\end{abstract}

PACS number(s); 03.67.-a, 03.67.Lx, 42.50.-p

\section{INTRODUCTION}

The theory of quantum computation promises to revolutionize the future of computer technology with merits in factoring large integers [1] and combinational searches [2]. In recent years, the physical implementation of a quantum computer has been intensively studied. Quantum computing in optical systems has been studied as one of several plausible models. Recently, Knill et al. suggested a scheme for efficient quantum computation with linear optics [3].

A coherent field is a fundamental tool in quantum optics and linear superposition of two coherent states is considered one of the realizable mesoscopic quantum systems [1]. In particular, Cochrane et al. [5] showed how logical qubits can be implemented using even and odd coherent superposition states which are defined as $N_{ \pm}^{0}(|\alpha\rangle \pm|-\alpha\rangle)$ with $|\alpha\rangle$ and $|-\alpha\rangle$ representing coherent states of $\pi$ phase difference and $N_{ \pm}^{0}$ being the normalization factors. The two superposition states form orthogonal bases in twodimensional Hilbert space and they can be discriminated by photon measurement [6]. There were some proposals to entangle such the logical qubits with atomic states [7]. One drawback of using even and odd cat states as a logical qubit basis for quantum computation is that they are extremely sensitive to photon loss and detection inefficiency.

In this paper, we present a method to implement universal quantum computation using coherent states. This proposal makes it possible to realize universal quantum computation based on quantum teleportation [8] which was shown to be a useful tool in controlled gate operation [9]. It is also found that this scheme is robust to detection inefficiency.

\section{READOUT SCHEME AND UNIVERSAL GATE OPERATIONS}

Let us consider two coherent states $|\alpha\rangle$ and $|-\alpha\rangle$, where the coherent amplitude $\alpha$ is taken to be real. The two coherent states are not orthogonal to each other but their overlap $\langle\alpha \mid-\alpha\rangle=\mathrm{e}^{-2 \alpha^{2}}$ decreases exponentially with $\alpha$. For example, when $\alpha$ is as small as 3, the overlap is $\approx 10^{-8}$. Throughout the paper, the average photon number of the coherent state is assumed around 10. We identify the two coherent states of $\alpha$ as basis states for a logical qubit:

$$
|\alpha\rangle \rightarrow\left|0_{L}\right\rangle, \quad|-\alpha\rangle \rightarrow\left|1_{L}\right\rangle .
$$

A qubit state is then represented by $|\phi\rangle=\mathcal{A}|\alpha\rangle+\mathcal{B}|-\alpha\rangle$ where the normalization condition is

$$
\begin{aligned}
1 & =\langle\phi \mid \phi\rangle=|\mathcal{A}|^{2}+|\mathcal{B}|^{2}+\left(\mathcal{A B}^{*}+\mathcal{A}^{*} \mathcal{B}\right)\langle\alpha \mid-\alpha\rangle \\
& \approx|\mathcal{A}|^{2}+|\mathcal{B}|^{2} .
\end{aligned}
$$

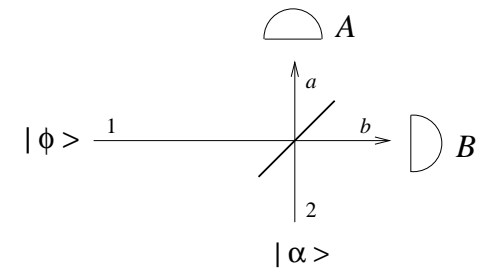

FIG. 1. Measurement scheme for $|\phi\rangle_{1}=\mathcal{A}|\alpha\rangle_{1}+\mathcal{B}|-\alpha\rangle_{1}$ with a 50-50 beam splitter and auxiliary state $|\alpha\rangle_{2}$. If detector $A$ registers any photon(s) while detector $B$ does not, the measurement outcome is $|\alpha\rangle$, i.e. $\left|0_{L}\right\rangle$. On the contrary, $A$ does not click while $B$ does, the measurement outcome is $|-\alpha\rangle$, i.e. $\left|1_{L}\right\rangle$.

Let us consider the readout of a qubit. The logical basis states, $|\alpha\rangle$ and $|-\alpha\rangle$, can be discriminated by a simple measurement scheme with a 50-50 beam splitter, an auxiliary coherent field of amplitude $\alpha$ and two photodetectors as shown in Fig. 1. At the beam splitter, the input state $|\phi\rangle_{1}$ is superposed with the auxiliary state $|\alpha\rangle_{2}$ and gives the output

$$
\left|\phi_{R}\right\rangle_{a b}=\mathcal{A}|\sqrt{2} \alpha\rangle_{a}|0\rangle_{b}+\mathcal{B}|0\rangle_{a}|-\sqrt{2} \alpha\rangle_{b} .
$$

If detector $A$ registers any photon(s) while detector $B$ does not, we know that $|\alpha\rangle$ is measured. On the contrary, if $A$ does not click while $B$ does, the measurement outcome is $|-\alpha\rangle$. Even though there is a non-zero probability of failure $P_{f}={ }_{a}\left\langle\left.\left. 0\right|_{b}\left\langle 0 \mid \phi_{R}\right\rangle_{a b}\right|^{2}=|\mathcal{A}+\mathcal{B}|^{2} \mathrm{e}^{-2 \alpha^{2}}\right.$ in which both of the detectors do not register a photon, the failure is known from the result whenever it occurs, and $P_{f}$ approaches to zero exponentially as $\alpha$ increases. 
(a)

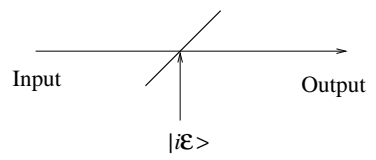

(b)
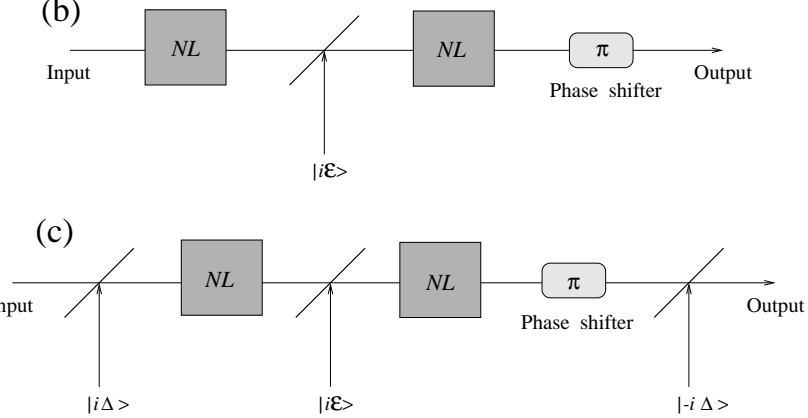

FIG. 2. 1-bit rotation around the $z$ (a), $y(\mathrm{~b})$, and $x$ axes (c) for a qubit state of coherent fields. $N L$ represents a nonlinear medium. The transmission coefficient $T$ of the beam splitters is assumed to be close to unity. $\mathcal{E}$ corresponds to $\frac{\theta}{4 \alpha \sqrt{1-T}}$, where $\theta$ is the required degree for a rotation and $\alpha$ is the coherent amplitude of the qubit state $|\phi\rangle . \Delta=\frac{\pi}{8 \alpha \sqrt{1-T}}$. Starting from a coherent state, an arbitrary qubit can be prepared up to a global phase using the above operations.

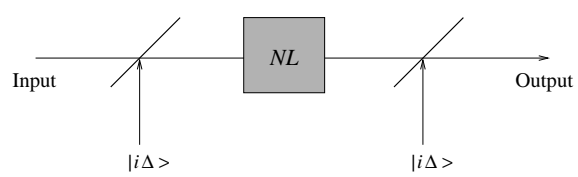

FIG. 3. Hadamard gate for a qubit state $|\phi\rangle=\mathcal{A}|\alpha\rangle+$ $\mathcal{B}|-\alpha\rangle$. The coherent field amplitude $i \Delta$ is $i \frac{\pi}{8 \alpha \sqrt{1-T}}$ and the transmission coefficient $T$ of the beam splitters is close to unity. The irrelevant global phase is neglected.

An arbitrary 1-bit rotation and a controlled-NOT (CNOT) gate for two-qubit states form a set which satisfies all the requirements for a universal gate operation. For any $\mathrm{SU}(2)$ unitary operation, there is a unique rotation $R(\theta, \phi, \eta)$ around the $x, y$ and $z$ axes. Cochrane et $a l$. showed that the rotation around $x$ axis for even and odd coherent superposition states can be realized using a interaction Hamiltonian $H_{D}=\hbar\left(\beta a^{\dagger}+\beta^{*} a\right)$, where $\beta$ is the complex amplitude of the classical driving force [5]. The evolution by this Hamiltonian corresponds to the displacement operator, $D(\delta)=\exp \left(\delta a^{\dagger}-\delta^{*} a\right)$, where $a$ and $a^{\dagger}$ are respectively annihilation and creation operators. In a similar way, $z$-rotation

$$
U_{z}(\theta / 2)=\left(\begin{array}{cc}
e^{i \theta / 2} & 0 \\
0 & e^{-i \theta / 2}
\end{array}\right)
$$

for a logical qubit $|\phi\rangle$ can be obtained. A coherent state is a displaced vacuum $|\alpha\rangle=D(\alpha)|0\rangle$. We know that two displacement operators $D(\alpha)$ and $D(\delta)$ do not commute but the product $D(\alpha) D(\delta)$ is simply $D(\alpha+\delta)$ multiplied by a phase factor, $\exp \left[\left(\alpha \delta^{*}-\alpha^{*} \delta\right) / 2\right]$. This phase factor plays a role to rotate the logical qubit. The action of displacement operator $D(i \epsilon)$, where $\epsilon(\ll \alpha)$ is real, on the qubit $|\phi\rangle$ is the same as the $z$-rotation of the qubit by $U_{z}(2 \alpha \epsilon)$. We can easily check their similarity by calculating the fidelity:

$$
\begin{aligned}
& \left|\left\langle\phi\left|U_{z}^{\dagger}(2 \alpha \epsilon) D(i \epsilon)\right| \phi\right\rangle\right|^{2} \\
& =e^{-\epsilon^{2}}\left\{|\mathcal{A}|^{2}+|\mathcal{B}|^{2}+e^{-2 \alpha^{2}}\left(\mathcal{A B}^{*} e^{-2 i \alpha \epsilon}+\mathcal{A}^{*} \mathcal{B} e^{2 i \alpha \epsilon}\right)\right\}^{2} \\
& \approx \exp \left[-\epsilon^{2}\right] \approx 1 .
\end{aligned}
$$

Thus the rotation angle $\theta$ depends on $\alpha$ and $\epsilon: \theta=4 \alpha \epsilon$. A small amount of $\epsilon$ suffices to make one cycle of rotation as $\alpha$ is relatively large. The displacement operation $D(i \epsilon)$ can be effectively performed using a beam splitter with the transmission coefficient $T$ close to unity and a high-intensity coherent field of amplitude $i \mathcal{E}$, where $\mathcal{E}$ is real, as shown in Fig. 2(a). It is known that the effect of the beam splitter is described by $D(i \mathcal{E} \sqrt{1-T})$ in the limit of $T \rightarrow 1$ and $\mathcal{E} \gg 1$. (More rigorously the output state becomes mixed but in the limit it can well be approximated to a pure state as shown by one of the authors [10].)

To achieve any arbitrary 1-bit rotation, we need to operate $U_{x}(\pi / 4)$ and $U_{x}(-\pi / 4)$ which are rotations by $\pi / 2$ and $-\pi / 2$, respectively, around the $x$ axis. We find that $U_{x}(\pi / 4)$ can be realized using a nonlinear medium. Even though the efficiency of nonlinear interaction can be a problem, there was an experimental report for a successful measurement of giant Kerr nonlinearity [11]. The anharmonic-oscillator Hamiltonian of an amplitudedispersive medium is 4

$$
\mathcal{H}_{N L}=\hbar \omega a^{\dagger} a+\hbar \Omega\left(a^{\dagger} a\right)^{2},
$$

where $\omega$ is the frequency of the coherent field and $\Omega$ is the strength of the anharmonic term. When the interaction time $t$ in the medium is $\pi / \Omega$, coherent states $|\alpha\rangle$ and $|-\alpha\rangle$ evolve as follows:

$$
\begin{aligned}
& |\alpha\rangle \longrightarrow \frac{e^{-i \pi / 4}}{\sqrt{2}}(|\alpha\rangle+i|-\alpha\rangle), \\
& |-\alpha\rangle \longrightarrow \frac{e^{-i \pi / 4}}{\sqrt{2}}(i|\alpha\rangle+|-\alpha\rangle) .
\end{aligned}
$$

This transformation corresponds to $U_{x}(\pi / 4)$ up to a global phase shift. The other rotation $U_{x}(-\pi / 4)$ can be realized by applying a phase shifter $P(\pi)$, which acts $|\alpha\rangle \leftrightarrow|-\alpha\rangle$, after or before $U_{x}(\pi / 4)$ operation. Note that $P(\pi)$ corresponds to $\pi$-rotation around the $x$ axis, i.e. a 1-bit NOT gate. The other two required unitary operations $U_{y}(\phi / 2)$ and $U_{z}(\eta / 2)$ which correspond to rotations around the $y$ and $x$ axes can be realized using the following identities [12]

$$
\begin{aligned}
& U_{y}(\phi / 2)=U_{x}(-\pi / 4) U_{z}(\phi / 2) U_{x}(\pi / 4), \\
& U_{x}(\eta / 2)=U_{z}(-\pi / 4) U_{y}(\eta / 2) U_{z}(\pi / 4) .
\end{aligned}
$$

Therefore, any 1-bit rotation can be performed up to a global phase with beam splitters, nonlinear media, phase 
shifters and auxiliary coherent light fields as shown in Fig. 2. As an example, we can construct the Hadamard gate $H$ as

$$
H=-U_{z}(\pi / 4) U_{x}(\pi / 4) U_{z}(\pi / 4),
$$

which is shown in Fig 3. Using these operations, any 1qubit state $|\phi\rangle=\mathcal{A}|\alpha\rangle+\mathcal{B}|-\alpha\rangle$ with arbitrary $\mathcal{A}$ and $\mathcal{B}$ can be prepared up to a global phase from a coherent state.

(a)

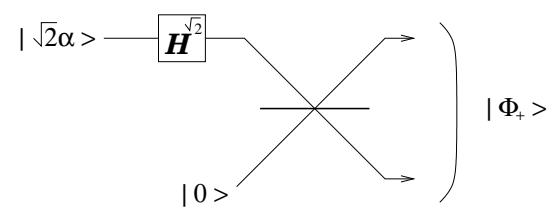

(b)

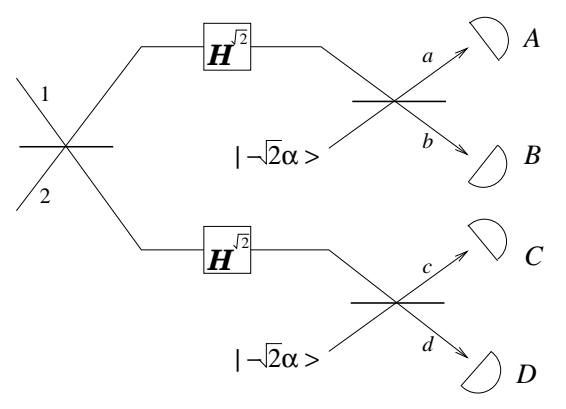

(c)

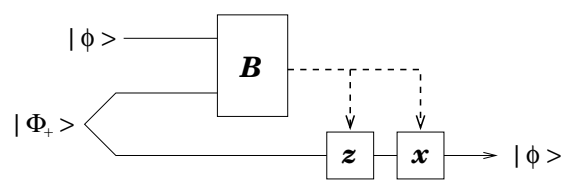

FIG. 4. Teleportation process for an unknown state $|\phi\rangle=\mathcal{A}|\alpha\rangle+\mathcal{B}|-\alpha\rangle . \quad H^{\sqrt{2}}$ represent the Hadamard gate with an incident qubit state of coherent amplitudes $\pm \sqrt{2} \alpha$. B represents the Bell measurement. $x$ and $z$ represent $\pi$ rotation around the $x$ and $z$ axes. (a) Generation of the quantum channel $\left|\Phi_{+}\right\rangle$. (b) Bell-state measurement with arbitrarily high precision. If detector $A$ does not click, the measurement outcome is $\left|\Phi_{+}\right\rangle$, and so on. Only one of the four detectors does not detect any photon at a measurement event for $\alpha \gg 1$. (c) Scheme to teleport $|\phi\rangle$ via the entangled quantum channel $\left|\Phi_{+}\right\rangle$. The Pauli operations represented by $x$ and $z$ are performed according to the result of Bell measurement $B$.

For a universal gate operation, a CNOT gate is required besides 1-bit rotation. It was found that the CNOT operation can be realized using a teleportation protocol [9]. For a superposition of coherent states, quantum teleportation protocols have been suggested by utilizing an entangled coherent state 13, 14, including an entanglement purification scheme 14]. However, the success probability of this teleportation scheme is limited to less than $1 / 2$ in practice and the required photon parity measurement is very sensitive to detection inefficiency and photon loss as the parity alternates by missing one photon. We suggest a teleportation protocol as follows to circumvent those problems. (a)

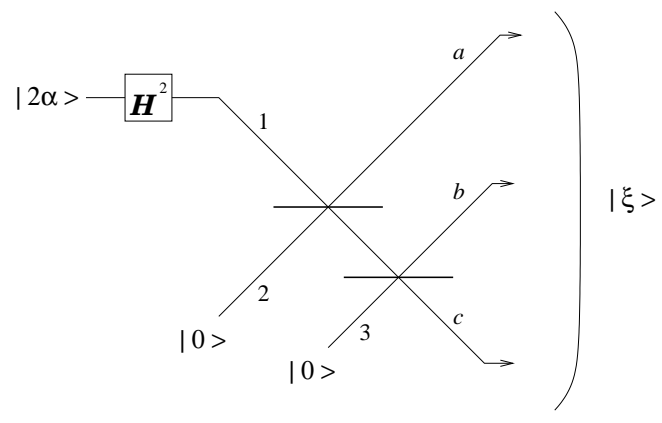

(b)

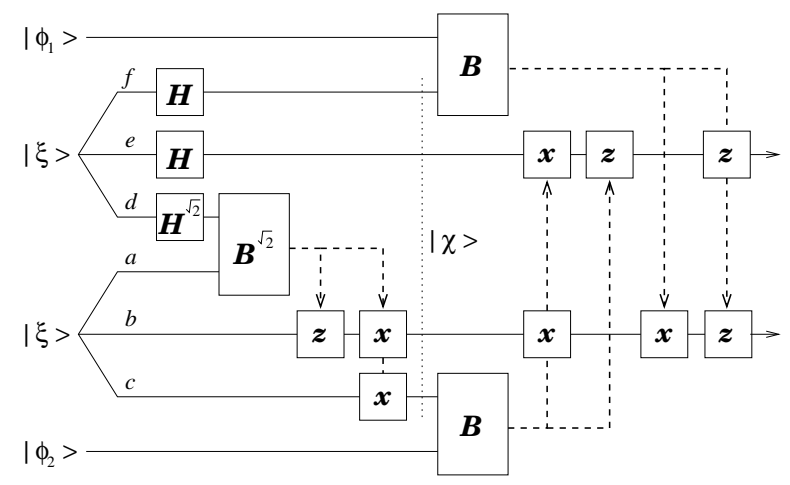

FIG. 5. CNOT operation using teleportation protocol and three-mode entanglement. (a) Generation of a three-mode entangled state $|\xi\rangle=\mathcal{N}(|\sqrt{2} \alpha, \alpha, \alpha\rangle+|-\sqrt{2} \alpha,-\alpha,-\alpha\rangle$ with beam splitters. $H^{2}$-gate is the Hadamard gate with an incident qubit state of amplitudes $\pm 2 \alpha$. (b) CNOT operation with the use of the coherent field $|\xi\rangle$ and the teleportation protocol. A four-mode entangled state $|\chi\rangle$ is generated for the operation at the left-hand side of the circuit. $\left|\phi_{1}\right\rangle$ is the target bit and $\left|\phi_{2}\right\rangle$ is the control bit here.

For any ideal teleportation scheme, a maximally entangled pair, Bell measurement and unitary operations are required [8]. In our case, necessary unitary operations $\sigma_{x}$ and $\sigma_{z}$ correspond to a phase shift $P(\pi)$ and displacement operation $D\left(\frac{i \pi}{4 \alpha \sqrt{1-T}}\right)$ respectively. An entangled coherent channel $\left|\Phi_{+}\right\rangle=\mathcal{N}_{+}(|\alpha\rangle|\alpha\rangle+|-\alpha\rangle|-\alpha\rangle)$, where $\mathcal{N}_{+}$ is a normalization factor, can be generated from a coherent state passing through a $H^{\sqrt{2}}$ gate and a $50-50$ beam splitter as shown in Fig. Q(a). The superscript $\sqrt{2}$ in $H^{\sqrt{2}}$ stands for the amplitude of the incident field being $\sqrt{2} \alpha$. Note that the coherent amplitude $i \Delta$ for a unitary operation shown in Fig. 3 should be $i \pi /[8 \alpha \sqrt{2(1-T)}]$ for the $H^{\sqrt{2}}$-gate operation. The Bell measurement shown in Fig. 1 (b) is to distinguish four quasi-Bell states [15],

$$
\begin{aligned}
& \left|\Phi_{ \pm}\right\rangle=\mathcal{N}_{ \pm}(|\alpha, \alpha\rangle \pm|-\alpha,-\alpha\rangle), \\
& \left|\Psi_{ \pm}\right\rangle=\mathcal{N}_{ \pm}(|\alpha,-\alpha\rangle \pm|-\alpha, \alpha\rangle),
\end{aligned}
$$

where $| \pm \alpha, \pm \alpha\rangle=| \pm \alpha\rangle \otimes| \pm \alpha\rangle$. Note that the quasi-Bell states become maximally entangled Bell states when $\alpha$ is large. If the incident field to the first beam splitter in Fig. 国 (b) is $\left|\Phi_{+}\right\rangle_{12}$, it becomes $|0,2 \alpha,-\sqrt{2} \alpha, \sqrt{2} \alpha\rangle_{a b c d}$ at detectors $A, B, C$, and $D$. If detector $A$ does not 
click while the others do, the measurement outcome is $\left|\Phi_{+}\right\rangle_{12}$. Likewise, only $B$ does not click for the measurement outcome $\left|\Phi_{-}\right\rangle_{12}, C$ for $\left|\Psi_{+}\right\rangle_{12}$, and $D$ for $\left|\Psi_{-}\right\rangle_{12}$. The failure probability for which no photon is detected at more than one detector, which is due to the non-zero probability of $\langle 0 \mid \pm 2 \alpha\rangle$ and $\langle 0 \mid \pm \sqrt{2} \alpha\rangle$, approaches to zero rapidly as $\alpha$ increases, and, moreover, the failure is always known when it occurs. The scheme to teleport $|\phi\rangle$ via the entangled channel $\left|\Phi_{+}\right\rangle$is summarized in Fig. $4(\mathrm{c})$. When the Bell measurement outcome is $\left|\Phi_{+}\right\rangle$, the output state does not need any operation. When the Bell measurement outcome is $\left|\Phi_{-}\right\rangle$or $\left|\Psi_{+}\right\rangle, \sigma_{z}$ or $\sigma_{x}$ is required respectively. The unitary operations $\sigma_{z}$ and $\sigma_{x}$ should be successively applied for the outcome $\left|\Psi_{-}\right\rangle$.

Gottesman and Chuang showed that the teleportation protocol can be used to construct a CNOT gate [9]. To apply their suggestion in our scheme, we need to use two three-mode entangled states represented by

$$
|\xi\rangle=\mathcal{N}(|\sqrt{2} \alpha, \alpha, \alpha\rangle+|-\sqrt{2} \alpha,-\alpha,-\alpha\rangle),
$$

where $\mathcal{N}$ is a normalization factor, and the quantum teleportation protocol we just developed. The entangled state $|\xi\rangle$ can be generated by passing a coherent field $|2 \alpha\rangle$ through a $H^{2}$-gate, which is a Hadamard gate for a qubit with logical bases $| \pm 2 \alpha\rangle$, and two 50-50 beam splitters as shown in Fig. 5(a). After generating $|\xi\rangle_{a b c}$ and $|\xi\rangle_{d e f}$, Hadamard operations are applied to $|\xi\rangle_{\text {def }}$ as shown in Fig. 5(a). This makes the given state $|\xi\rangle_{a b c} \otimes|\xi\rangle_{\text {def }}$ to be

$$
\begin{aligned}
& \longrightarrow\left|\Phi_{+}^{\prime}\right\rangle_{a d}\{|\alpha, \alpha\rangle(|\alpha, \alpha\rangle+|-\alpha,-\alpha\rangle) \\
&+|-\alpha,-\alpha\rangle(|\alpha,-\alpha\rangle+|-\alpha, \alpha\rangle)\}_{b c e f} \\
&\left|\Phi_{-}^{\prime}\right\rangle_{a d}\{|\alpha, \alpha\rangle(|\alpha, \alpha\rangle+|-\alpha,-\alpha\rangle) \\
&-|-\alpha,-\alpha\rangle(|\alpha,-\alpha\rangle+|-\alpha, \alpha\rangle)\}_{b c e f} \\
&\left|\Psi_{+}^{\prime}\right\rangle_{a d}\{|-\alpha,-\alpha\rangle(|\alpha, \alpha\rangle+|-\alpha,-\alpha\rangle) \\
&+|\alpha, \alpha\rangle(|\alpha,-\alpha\rangle+|-\alpha, \alpha\rangle)\}_{b c e f} \\
&\left|\Psi_{-}^{\prime}\right\rangle_{a d}\{|-\alpha,-\alpha\rangle(|\alpha, \alpha\rangle+|-\alpha,-\alpha\rangle) \\
&-|\alpha, \alpha\rangle(|\alpha,-\alpha\rangle+|-\alpha, \alpha\rangle)\}_{b c e f}
\end{aligned}
$$

where $\left|\Phi_{ \pm}^{\prime}\right\rangle$ and $\left|\Psi_{ \pm}^{\prime}\right\rangle$ are quasi-Bell states with the coherent amplitude $\pm \sqrt{2} \alpha$ and the normalization factor is omitted. The Bell measurement $B^{\sqrt{2}}$ in the figure, must be performed on modes $a$ and $d$. It can be easily shown from Eq. (15) that a four-mode entangled state

$$
\begin{aligned}
&|\chi\rangle_{\text {bce }}=\mathcal{N}^{\prime} {[|\alpha, \alpha\rangle(|\alpha, \alpha\rangle+|-\alpha,-\alpha\rangle)} \\
&+|-\alpha,-\alpha\rangle(|\alpha,-\alpha\rangle+|-\alpha, \alpha\rangle)],
\end{aligned}
$$

where $\mathcal{N}^{\prime}$ is a normalization factor, is generated after the appropriate unitary operation according to the Bell measurement result as shown in Fig. 5(b). The entangled state $|\chi\rangle_{\text {bcef }}$ is used to complete the CNOT gate on the right-hand side of the circuit in Fig. 5(b), which can be verified by a little algebra [9].

\section{ESTIMATION OF POSSIBLE ERRORS}

We have shown that universal quantum computation using coherent states can be realized using coherent states. We already pointed out that the failure probability for the measurement which is of the order of $|\langle\sqrt{2} \alpha \mid 0\rangle|^{2}$ is not only very small for a reasonably large $\alpha$ but also the failure is known whenever it occurs. If the detection efficiency of a photodetector is $d$, the failure probability $P_{f}^{d}$ of the detector not to register any photon, while the incident field is $\left|\phi_{R}\right\rangle_{a b}$ in Eq. (3), is

$$
\begin{aligned}
P_{f}^{d} & =\sum_{n, m=0}^{\infty} \mid{ }_{a}\left\langle\left.\left. n\right|_{b}\left\langle m \mid \phi_{R}\right\rangle_{a b}\right|^{2}(1-d)^{n}(1-d)^{m}\right. \\
& \approx \sum_{n=0}^{\infty}|\langle n \mid \sqrt{2} \alpha\rangle|^{2}(1-d)^{n}
\end{aligned}
$$

where approximation (2) is used. For example, suppose that $\alpha=3$ and the detection efficiency of the detectors is $90 \%$ which is a reasonable value for an avalanched photodetector [16], the failure probability $P_{f}^{d}$ that the detector misses all the photons is $P_{f}^{d} \approx 9 \times 10^{-8}$.

If the effect of $\epsilon$ for the displacement operator is not negligible, a qubit state $\left|\phi^{\prime}\right\rangle_{1}=D\left(i \epsilon_{1}\right) \cdots D\left(i \epsilon_{N}\right)|\phi\rangle_{1}$ after $N$ displacement operations may be

$$
\left|\phi^{\prime}\right\rangle_{1}=\mathcal{A}^{\prime}\left|\alpha+i \sum_{n=1}^{N} \epsilon_{n}\right\rangle_{1}+\mathcal{B}^{\prime}\left|-\alpha+i \sum_{n=1}^{N} \epsilon_{n}\right\rangle_{b}
$$

After passing a 50-50 beam splitter with an auxiliary state $|\alpha\rangle_{2}$ as shown in Fig. $\mathbb{1}$, the state $\left|\phi^{\prime}\right\rangle_{1}$ becomes

$$
\begin{aligned}
\left|\phi_{R}^{\prime}\right\rangle_{a b}=\mathcal{A}^{\prime} \mid \sqrt{2} \alpha & \left.+\frac{i}{\sqrt{2}} \sum_{n=1}^{N} \epsilon_{n}\right\rangle_{a}\left|\frac{i}{\sqrt{2}} \sum_{n=1}^{N} \epsilon_{n}\right\rangle_{b} \\
& +\mathcal{B}^{\prime}\left|\frac{i}{\sqrt{2}} \sum_{n=1}^{N} \epsilon_{n}\right\rangle_{a}\left|-\sqrt{2} \alpha+\frac{i}{\sqrt{2}} \sum_{n=1}^{N} \epsilon_{n}\right\rangle_{b} .
\end{aligned}
$$

In this condition, there is non-zero probability $\widetilde{P}_{f}^{d}$ for undetected errors in which detector $A(B)$ detects any photon and $B(A)$ does not while the incident state $\left|\phi^{\prime}\right\rangle_{1}$ was $\left|1_{L}\right\rangle\left(\left|0_{L}\right\rangle\right)$ (see Fig. 11). For the worst case, all $\epsilon_{n}$ 's may have the same sign with a large $N$. One useful trick to overcome this problem is to flip the sign of $\epsilon_{n}$ appropriately for each operation, noting that the rotation $R_{z}(\theta)$ can be performed both by positive and negative $\theta$. By this way, we can keep $\sum_{n=1}^{N} \epsilon_{n} \sim \bar{\epsilon}=\pi / 4 \alpha$, regardless of $N$, then Eq. (19) can be represented as 


$$
\begin{aligned}
\left|\phi_{R}^{\prime}\right\rangle_{a b}= & \mathcal{A}^{\prime}\left|\sqrt{2} \alpha+\frac{i \bar{\epsilon}}{\sqrt{2}}\right\rangle_{a}\left|\frac{i \bar{\epsilon}}{\sqrt{2}}\right\rangle_{b} \\
& +\mathcal{B}^{\prime}\left|\frac{i \bar{\epsilon}}{\sqrt{2}}\right\rangle_{a}\left|-\sqrt{2} \alpha+\frac{i \bar{\epsilon}}{\sqrt{2}}\right\rangle_{b}
\end{aligned}
$$

In this condition, the fidelity between the final state (18) and the ideal output is proportional to $e^{-\epsilon^{2}}$ from Eq. (5). Fidelity of $\approx 0.93$ is then obtained for $\alpha=3$.

Differently from $P_{f}^{d}$, the undetected error probability $\widetilde{P}_{f}^{d}$ is a probability of making an error without being recognized. Considering the accumulated error as in Eq. (20), in order to minimize the undetected error $\widetilde{P}_{f}^{d}$ while keeping $P_{f}^{d}$ low, we need to modify the criterion to discriminate $| \pm \sqrt{2} \alpha+i \bar{\epsilon} / \sqrt{2}\rangle$ and $|i \bar{\epsilon} / \sqrt{2}\rangle$. Ideally we took $\bar{\epsilon}=0$ and discriminated the two states by detection of any photons and no photon. In this case, the probability of $| \pm \sqrt{2} \alpha+i \bar{\epsilon} / \sqrt{2}\rangle$ registering no photon is

$$
p_{A}=\sum_{n=0}^{\infty}|\langle n \mid \pm \sqrt{2} \alpha+i \bar{\epsilon} / \sqrt{2}\rangle|^{2}(1-d)^{n}
$$

and the probability of the state $|i \bar{\epsilon} / \sqrt{2}\rangle$ registering one or more photons is

$$
p_{B}=\sum_{m=1}^{\infty} \sum_{n=m}^{\infty}|\langle n \mid i \bar{\epsilon} / \sqrt{2}\rangle|^{2}{ }_{n} C_{m} d^{m}(1-d)^{n-m}
$$

where ${ }_{n} C_{m}=n ! / m !(n-m)$ !. Both $p_{A}$ and $p_{B}$ approach to zero as $\alpha$ increases. We then obtain undetected error probability $\widetilde{P}_{f}^{d}=p_{A} \times p_{B}$. On the other hand, the success probability $P_{s}$ is the probability in that $|i \bar{\epsilon} / \sqrt{2}\rangle$ yields no photon and $| \pm \sqrt{2} \alpha+i \bar{\epsilon} / \sqrt{2}\rangle$ yields any photon(s):

$$
\begin{aligned}
& P_{s}=\sum_{n=0}^{\infty}|\langle n \mid i \epsilon / \sqrt{2}\rangle|^{2}(1-d)^{n} \times \\
& \sum_{m=1}^{\infty} \sum_{n=m}^{\infty}|\langle n \mid \sqrt{2} \alpha+i \bar{\epsilon} / \sqrt{2}\rangle|^{2}{ }_{n} C_{m} d^{m}(1-d)^{n-m} .
\end{aligned}
$$

The detected error probability is $P_{f}^{d}=1-P_{s}-\widetilde{P}_{f}^{d}$. Suppose that $\alpha=3(\bar{\epsilon}$ is then $\approx 0.26)$, and the detection efficiency is again $90 \%, p_{A} \approx 9 \times 10^{-8}$ and $p_{B} \approx 0.030$ are obtained. If we keep the criterion for the ideal case, we find $\widetilde{P}_{f}^{d} \approx 3 \times 10^{-9}$ and $P_{f}^{d} \approx 0.030$. However, if we take the registration of 0,1 and 2 photons as the measurement of $|i \bar{\epsilon} / \sqrt{2}\rangle$ then $p_{A}, p_{B}$ and $P_{s}$ should be re-defined as follows:

$$
\begin{aligned}
p_{A}= & \sum_{n=0}^{\infty}|\langle n \mid \sqrt{2} \alpha+i \bar{\epsilon} / \sqrt{2}\rangle|^{2}(1-d)^{n} \\
& +\sum_{n=1}^{\infty}|\langle n \mid \sqrt{2} \alpha+i \bar{\epsilon} / \sqrt{2}\rangle|^{2} d(1-d)^{n-1} \\
& +\sum_{n=2}^{\infty}|\langle n \mid \sqrt{2} \alpha+i \bar{\epsilon} / \sqrt{2}\rangle|^{2} d^{2}(1-d)^{n-2}
\end{aligned}
$$

$$
\begin{aligned}
p_{B}= & \sum_{m=3}^{\infty} \sum_{n=m}^{\infty}|\langle n \mid i \bar{\epsilon} / \sqrt{2}\rangle|^{2}{ }_{n} C_{m} d^{m}(1-d)^{n-m} \\
P_{s}= & \left\{\sum_{n=0}^{\infty}|\langle n \mid i \bar{\epsilon} / \sqrt{2}\rangle|^{2}(1-d)^{n}\right. \\
& +\sum_{n=1}^{\infty}|\langle n \mid i \bar{\epsilon} / \sqrt{2}\rangle|^{2} d(1-d)^{n-1} \\
& \left.+\sum_{n=2}^{\infty}|\langle n \mid i \bar{\epsilon} / \sqrt{2}\rangle|^{2} d^{2}(1-d)^{n-2}\right\} \times \sum_{m=3}^{\infty} \sum_{n=m}^{\infty} \\
& |\langle n \mid \sqrt{2} \alpha+i \bar{\epsilon} / \sqrt{2}\rangle|^{2}{ }_{n} C_{m} d^{m}(1-d)^{n-m} .
\end{aligned}
$$

We then find $\widetilde{P}_{f}^{d} \approx 6 \times 10^{-11}$ and $P_{f}^{d} \approx 2 \times 10^{-5}$ for $\alpha=3$ and $d=0.9$. Recently, Takeuchi et al. [16] developed an avalanched photodetector which can discern 0,1 , and 2 photons with high efficiency.

Decoherence is considered one of the main obstacles in quantum computation. When a qubit state $|\phi\rangle$ is subject to a vacuum environment it evolves to 17

$$
\begin{aligned}
\rho_{M}(\tau)=\mathcal{N}_{\tau} & \left\{|\mathcal{A}|^{2}|t \alpha\rangle\left\langle\left. t \alpha|+| \mathcal{B}\right|^{2} \mid-t \alpha\right\rangle\langle-t \alpha|\right. \\
+ & \left.\Gamma\left(\mathcal{A} \mathcal{B}^{*}|t \alpha\rangle\left\langle-t \alpha\left|+\mathcal{A}^{*} \mathcal{B}\right|-t \alpha\right\rangle\langle t \alpha|\right)\right\}
\end{aligned}
$$

where $t=e^{-\gamma \tau / 2}, \Gamma=e^{-2\left(1-t^{2}\right) \alpha^{2}}, \gamma$ is the energy decay rate, $\tau$ is the interaction time, and $\mathcal{N}_{\tau}$ is the normalization factor. Considering decoherence, we need to change $\left|0_{L}\right\rangle$ and $\left|1_{L}\right\rangle$ to $|t \alpha\rangle$ and $|-t \alpha\rangle$. The auxiliary coherent fields for computation have to be changed likewise. The larger the initial coherent amplitude $\alpha$ is, the longer the condition that $\langle t \alpha \mid-t \alpha\rangle \approx 0$ is preserved, but the decoherence becomes more rapid as $\alpha$ increases because $\Gamma$ decreases more rapidly for a larger $\alpha$. The energy decay rate $\gamma$ of the relevant system and number of required operations for computation may be the crucial factors to decide the value of $\alpha$. However, decohered states can still be represented by combinations of 1-bit errors for timedependent logical qubits $|t \alpha\rangle$ and $|-t \alpha\rangle$. It is known that an error correction circuit for an arbitrary 1-qubit error can be built using CNOT and 1-bit unitary operations |18.

\section{REMARKS}

In conclusion, we have found that near-deterministic universal quantum computation can be realized using coherent states. Efficient readout is possible using beam splitters and coherent light sources. Single-bit unitary transformation can be performed using beam splitters and nonlinear media, and CNOT gate can be constructed based on teleportation protocol. Teleportation of a coherent state qubit can be accomplished with a complete Bell measurement for a large coherent amplitude using nonlinear media, photodetectors, coherent light sources, 
and beam splitters. Decohered states can be represented by combinations of 1-bit errors for time-dependent coherent state qubits of reduced amplitude. A purification scheme for decohered entangled channels has been studied [19]. Detailed error correction methods for our scheme deserves further investigation. The nonlinear effect [4] used in this paper is typically too weak to generate required superposition states in current technology. The study of generating coherent superposition of optical states requires further study.

\section{ACKNOWLEDGMENTS}

We thank the UK Engineering and Physical Sciences Research Council for financial support through GR/R33304. HJ acknowledges the Overseas Research Student award.

[1] P. Shor, in Proceedings of the 35th Annual Symposium on Foundation of Computer Science (IEEE Computer Society Press, Santa Fe, NM, 1994).

[2] L. K. Grover, Phys. Rev. Lett. 79, 325 (1997).

[3] E. Knill, R. Laflamme, and G. J. Milburn, Nature 409, 46 (2001).

[4] B. Yurke and D. Stoler, Phys. Rev. Lett. 57, 13 (1986).

[5] P. T. Cochrane, G. J. Milburn, and W. J. Munro, Phys. Rev. A 59, 2631 (1999).

[6] V. Bužek and P. L. Knight, in Progress in Optics XXXIV, edited by E. Wolf (Elsevier, Amsterdam, 1995).

[7] W. J. Munro, G. J. Milburn, and B. C. Sanders, Phys. Rev. A 62, 052108 (2000); M. C. de Oliveira and W. J. Munro, Phys. Rev. A. 61, 042309 (2000).

[8] C. H. Bennett, G. Brassard, C. Crépeau, R. Jozsa, A. Peres, and W. K. Wootters, Phys. Rev. Lett. 70, 1895 (1993).

[9] D. Gottesman and I. L. Chuang, Nature 402, 390 (1999).

[10] M. S. Kim, G. Antesberger, C. T. Bodendorf and H. Walther, Phys. Rev. A 58, R65 (1998); M. S. Kim and J. Lee Phys. Rev. A 64, 012309 (2001).

[11] L. V. Hau, S. E. Harris, Z. Dutton, and C. H. Behroozi, Nature 397, 594 (1999).

[12] G. B. Arfken, Mathematical Method for Physicists, 3rd edition, p257 (Academic, New York, 1985).

[13] S. J. van Enk and O. Hirota, Phys. Rev. A 64, 022313 (2001).

14] H. Jeong, M. S. Kim, and J. Lee, e-print quantph/0104090, to be published in Phys. Rev. A.

[15] O. Hirota and M. Sasaki, e-print quant-ph/0101018.

[16] S. Takeuchi, Y. Yamamoto and H. H. Hogue, Appl. Phys. Lett. 74, 1063 (1999).

[17] S. J. D. Phoenix, Phys. Rev. Lett. 41, 5132 (1990).

[18] R. Laflamme, C. Miquel, J. P. Paz, and W. H. Zurek, Phys. Rev. Lett. 77, 198 (1996).
[19] H. Jeong and M. S. Kim, e-print quant-ph/0111015. 